\title{
Editorial: Does Research on Foundations of Quantum Mechanics Fit into PRX's Scope?
}

Physical Review X (PRX) is a broad-scope journal that aims to publish a limited number of selected best papers from all areas of physics: pure, applied, and interdisciplinary. So, when we are asked the question, "Does research on foundations of quantum mechanics fit into PRX's scope?" our answer must be a clear "yes." However, that is far from saying that any intellectually sound paper from that topical area merits a spot in PRX. Indeed, the task of judging whether an individual paper on foundations of quantum mechanics is suitable for PRX is an editorially challenging one, especially given the often unavoidable presence of philosophy in our efforts to understand, interpret, and apply quantum mechanics.

The paper by Hall, Deckert, and Wiseman [Phys. Rev. X, 4, 041013 (2014)] has given us a concrete editorial exercise to address this challenge. During the review process for the paper, we sought from both its authors and referees their thoughts on what kind of papers from this topical area should be considered suitable for PRX, and we had our own discussions. In the end, we came away with the following guideline: PRX will publish papers on foundations of quantum mechanics proposing highly original, scientifically sound approaches or models that already, or can potentially, suggest new experiments, and/or lead to new numerical tools for solving quantum-mechanical problems, or even significant new predictions. By applying this general guideline to the paper by Hall, Deckert, and Wiseman based on the information we gathered through its review process, we believed that it deserved publication in PRX.

And we have invited a Commentary by Bill Poirier from Texas Tech University that we hope will enhance your understanding of the paper and of our decision to publish it.

The Editors

\section{The Many Interacting Worlds Approach to Quantum Mechanics}

Bill Poirier , Department of Chemistry and Biochemistry, and Department of Physics, Texas Tech University, Box 41061, Lubbock, Texas 79409-1061

A Commentary on:

Quantum Phenomena Modeled by Interactions between Many Classical Worlds

Michael J. W. Hall, Dirk-André Deckert, and Howard M. Wiseman

Phys. Rev. X, 4, 041013 (2014)

Valid interpretations of quantum mechanics are like analytic solutions of the Schrödinger equation itself: not so many are known, but each one provides tremendous insight—and very often also practical benefits. For example, the many worlds (MW) interpretation [1], with its elegant treatment of apparent wave function "collapse," set the stage for applications such as decoherence, quantum computing, and quantum information that are now at the forefront of topical interest. Similarly, the de Broglie-Bohm (dBB) pilot wave interpretation [2] has inspired a recent flurry of quantum trajectory methods in the computational chemistry field. This is reason enough to take an interest in the "many interacting worlds" (MIW) approach, particularly as developed in the article by Hall et al. [3], but there are also other reasons.

To begin with, there is no wave function in the MIW approach, only trajectories. While this feature is shared with an earlier, continuous MIW interpretation of quantum mechanics [4-7], Hall et al. demonstrate that their discrete MIW is not only an "interpretation" per se but also an approach - meaning that its experimental predictions may differ from those of the Schrödinger equation. Hall et al. also introduce a key innovation with their "world-particle"- treating each trajectory world as if it were a single particle, and thence treating all quantum effects via a purely classical "interworld potential." The Schrödinger equation is thereby replaced by classical-like Hamiltonian equations of motion. These are great ideas-not only conceptually, but also with regard to the new numerical breakthroughs they are almost certain to engender.

\footnotetext{
*Bill.Poirier@ttu.edu
} 
Finally, there are important interpretational ramifications-which might be called "advantages," although any such assessment is admittedly subjective. In MW, for instance, the "worlds" are not rigorously defined (Everett himself spoke of the "universal wave function," not of many worlds per se [1].) In MIW, particularly the Hall et al. version, the worlds are built into the formalism right from the start. MW worlds, though noninteracting, perpetually branch in a manner that can be difficult to interpret precisely. In contrast, MIW worlds never branch or merge, but remain distinct throughout time. Moreover, MIW worlds do indeed interactthereby giving rise, in fact, to all quantum effects.

It is also interesting to compare MIW with $\mathrm{dBB}$. Both use quantum trajectories, but in $\mathrm{dBB}$ there is also a pilot wave. This hybrid ontology — wherein Nature not only behaves like both a particle and a wave, but actually is both-is sometimes regarded as a drawback. Moreover, the quantum trajectory is not a mathematical necessity in $\mathrm{dBB}$; it is built on top of the usual wave function structure, and is therefore arguably redundant. Finally, of the many quantum trajectories that could be physically real in $\mathrm{dBB}$, how does Nature decide which is the correct one? All of these issues disappear in the MIW approach; the wave function is dispensed with altogether and replaced with an ensemble of quantum trajectories, each of which is equally dynamically necessary and ontologically valid. Of course, the philosophical cost to be paid for this is the existence of many worlds.

Regardless of one's views with respect to a many-worlds ontology, the bigger point is simply this: practical benefits often follow from new ways of looking at things, and the MIW approach as developed by Hall et al. promises to be no exception.

About the Commentary author:

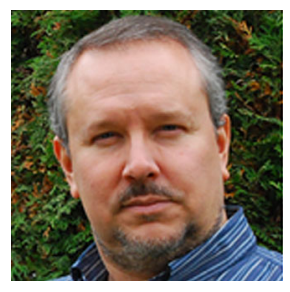

Bill Poirier is Chancellor's Council Distinguished Research Professor and also Barnie E. Rushing Jr. Distinguished Faculty Member at Texas Tech University, in the Department of Chemistry and Biochemistry and also the Department of Physics. He received his Ph.D. in theoretical physics from the University of California, Berkeley, followed by a chemistry research associateship at the University of Chicago. His research interest is in understanding and solving the Schrödinger equation, from both foundational and practical perspectives.

[1] H. Everett III, Rev. Mod. Phys. 29, 454 (1957).

[2] D. Bohm, Phys. Rev. 85, 166 (1952).

[3] Michael J. W. Hall, Dirk-André Deckert, and Howard M. Wiseman, Phys. Rev. X 4, 041013 (2014).

[4] P. Holland, Ann. Phys. (Berlin) 315, 505 (2005).

[5] B. Poirier, Chem. Phys. 370, 4 (2010).

[6] J. Schiff and B. Poirier, J. Chem. Phys. 136, 031102 (2012).

[7] B. Poirier, arXiv:1208.6260.

Published 23 October 2014

DOI: $10.1103 /$ PhysRevX.4.040002 